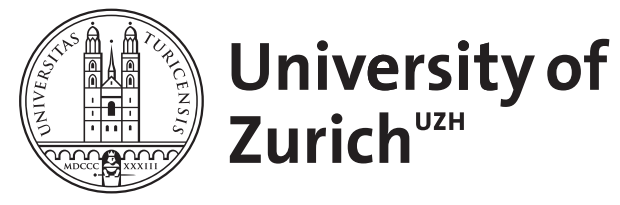

Zurich Open Repository and Archive

University of Zurich

University Library

Strickhofstrasse 39

CH-8057 Zurich

www.zora.uzh.ch

The evolution of costly mate choice against segregation distorters

Manser, Andri ; Lindholm, Anna K ; Weissing, Franz J

DOI: https://doi.org/10.1111/evo.13376

Posted at the Zurich Open Repository and Archive, University of Zurich

ZORA URL: https://doi.org/10.5167/uzh-144332

Journal Article

Accepted Version

Originally published at:

Manser, Andri; Lindholm, Anna K; Weissing, Franz J (2017). The evolution of costly mate choice against segregation distorters. Evolution, 71(12):2817-2828.

DOI: https://doi.org/10.1111/evo.13376 


\title{
The Evolution of Costly Mate Choice against Segregation Distorters
}

\author{
Andri Manser ${ }^{1,2, \star}$, Anna K. Lindholm ${ }^{3}$, Franz J. Weissing ${ }^{1}$ \\ 1 Theoretical Biology Group, Groningen Institute of Evolutionary Life Sciences \\ (GELIFES), University of Groningen, Groningen, The Netherlands \\ 2 Institute of Integrative Biology, Biosciences Building, University of Liverpool, \\ Liverpool, United Kingdom \\ ${ }^{3}$ Institute of Evolutionary Biology and Environmental Studies (IEU), University of \\ Zürich, Zürich, Switzerland \\ ^E-mail: Andri.Manser@liverpool.ac.uk
}

\begin{abstract}
1 Abstract
2 The evolution of female preference for male genetic quality remains a controversial 3 topic in sexual selection research. Conventional genetic mechanisms are usually in4 sufficient to maintain variation in male quality. As a consequence, benefits of a costly 5 choice become negligible- a problem known as the lek paradox. Here, we theoret6 ically investigate a scenario where females pay a direct fitness cost to avoid males 7 carrying an autosomal segregation distorter. We show that preference evolution is 8 greatly facilitated under such circumstances. Because the distorter is transmitted in 9 a non-Mendelian fashion, it can be maintained in the population despite directional 10 sexual selection. The preference helps females avoid fitness costs associated with the 11 distorter. Interestingly, we find that preference evolution is limited if the choice allele induces a very strong preference or if distortion is very strong. Moreover, the 3 preference can only persist in the presence of a signal that reliably indicates a male's distorter genotype. Hence, even in a system where the lek paradox does not play a


Directional sexual selection through female mate choice is likely to deplete genetic variation in male traits. If this occurs, genetic benefits of being choosy become small. This raises a simple yet puzzling question: why are females choosy if this choosiness depletes genetic variation in the male traits, which in turn is a prerequisite for the evolution of female choice? This fascinating question, generally known as the 'lek paradox', is a long-standing puzzle in sexual selection research (Kirkpatrick and Ryan, 1991).

Any resolution of this problem requires an explanation of how male trait variation persists despite directional sexual selection imposed by female choice. Several such explanations have been provided elsewhere (Pomiankowski et al., 1991; Pomiankowski and Moller, 1995; Kotiaho et al., 2001; Tomkins et al., 2004). Here, we want to theoretically examine the potential of segregation distorter systems to facilitate the evolution of costly female mate choice. By distorting transmission ratio in their own favor, distorters may act as generators of allelic variation in the male trait. In consequence, genetic variance in the trait may be maintained despite directional sexual selection. Moreover, distorters are usually associated with substantial fitness costs to their carriers (Burt and Trivers, 2006). Females may hence protect their offspring from detrimental fitness effects by avoiding fertilization with distorter-carrying males.

Connections between female choice and segregation distorters have been suggested by many empirical studies (see Wedell (2013) for a recent review). Female choice may happen both at a pre- and postmating stage. Premating preferences for an absence of distorters or for drive suppressors have been reported in stalk-eyed flies (Wilkinson et al., 1998; Cotton et al., 2014), house mice (Lenington et al., 1992), and Drosophila paulistorum (Miller et al., 2010). A larger body of work highlights the importance of mating biases at the postmating stage. As a direct consequence of segregation distortion, distorter carrying males are typically weak sperm competitors (Zeh and Zeh, 1997). Hence, female multiple mating (polyandry) has been proposed as a possible female counterstrategy against distorters (Haig and Bergstrom, 1995). Polyandry will lead to systematic deviations from random mating assumptions. It has thus been considered a form of indirect female mate choice (Brooks and Griffith, 2010). Evidence for distorters favouring polyandry ${ }^{\mathrm{c} 1}$ has been found in Drosophila simulans (Atlan ${ }^{c 1}$, to only name a few, have et al., 2004), Drosophila psendoobscura (Price et al., 2008), and the butterfly Hypolimnas bolina (Charlat et al., 2007). 
Given this considerable body of empirical evidence, surprisingly few studies have investigated the theoretical implications of segregation distortion on mating preferences. However, sexual selection models are complicated considerably when a distorter is added. While most population genetics models of sexual selection are framed in terms of haploids (Kuijper et al., 2012), segregation distortion requires the analysis of diploid organisms, which makes analysis much more intricate (Greenspoon and Otto, 2009). Most previously published models focus on the interplay between female choice and sex-linked distorters. Motivated by the stalk-eyed fly system (Wilkinson et al., 1998), two models investigated possible interactions between female choice and a sex-linked distorter. Reinhold et al. (1999) consider female choice for a distortion suppressor. The model suggests that, unexpectedly, female preferences in favour of a distortion suppressor is always selected against. Lande and Wilkinson (1999) chose a more direct approach and analyzed a situation where females choose a male trait (eyespan in this particular example) that indicates the absence of the distorter allele. They found that female preference for the trait can evolve, but only if the trait is perfectly coupled with the distorter. Even a small rate of recombination between a trait locus and the distorter locus will prevent the evolution of female choice. Randerson et al. (2000) investigated the evolution of costly male mate choice in the butterfly Acraea encedon infected with male-killing Wolbachia. Because the male killer causes a strong female bias in infected populations, sex-roles appear reversed and males should avoid infected females. The model confirms this expectation, as long as males do not perfectly discriminate between infected and uninfected females. In this case, costly male choice can stably persist. If males make no mistakes, costly male choice succumbs to its own success, since by effectively removing the male killer from the population, it also removes the benefits of being choosy.

Here, we investigate a model for the evolution of a costly female preference in the presence of an autosomal segregation distorter. In particular, we address the following questions: (1) Can the presence of an autosomal distorter facilitate the spread of a costly female preference for Mendelian segregation (i.e. distorter-free males)? (2) What levels of preference cost, preference and distortion strength allow for the evolution of costly female preferences? (3) How does recombination between a male sexual signal and a distorter affect evolutionary outcomes? 


\section{The Model}

Our model follows the standard set-up of population genetic models of sexual selection (Kuijper et al., 2012) and adds segregation distortion as an additional factor. We consider diploid organisms and three autosomal loci: a trait locus $T$ encoding for a sexual ornament in males; a preference locus $P$ affecting female choice for the ornament; and a distorter locus $S$ affecting Mendelian segregation in males. The following two alleles segregate at each of the three loci (see Table 1 for an overview).

- The trait locus $(T)$ is expressed in males only and encodes a trait that is subject to both viability and sexual selection. It contains alleles $T_{0}$ and $T_{1}$ (at frequencies $t_{0}$ and $t_{1}$, respectively), where allele $T_{1}$ induces a viability disadvantage but can be the target of female preference.

- The preference locus $(P)$ is expressed in females only and determines her relative tendency to mate with males of the three possible genotypes at the $T$ locus. It contains alleles $P_{0}$ and $P_{1}$ (at allele frequencies $p_{0}$ and $p_{1}$, respectively). The expression of female preference is associated with a fixed viability cost.

- The distorter locus $(S)$ contains alleles $S_{0}$ and $S_{1}$ (at allele frequencies $s_{0}$ and $s_{1}$, respectively). The proportion of distorter alleles $S_{1}$ transmitted to the next generation in $S_{0} S_{1}$ heterozygote males is given by parameter $d$, ranging from $d=0.5$ (Mendelian segregation) to $d=1$ (complete distortion). Fitness effects of the distorter are inspired by the $t$ haplotype system in house mice, where -depending on the distorter type $-S_{1} S_{1}$ homozygotes suffer either from male sterility (sterile type) or lethality in both sexes (lethal type).

The life cycle We consider an infinite population of diploids with non-overlapping generations. Because males and females are differently affected by selection, we track their genotype frequencies independently. Let $X_{i j, k l, m n}$ denote female genotype frequencies, where $i j$ defines status at the $T$ locus, $k l$ status at the $P$ locus, and $m n$ status at the distorter locus $S$. Analogously, male genotype frequencies are given by $Y_{o p, q r, s t}$. To derive the recursion equations for the resulting 64 ordered male and female genotypes, we assume the following life cycle.

We start our life cycle with the zygotes of the present generation. Analogous to above, the sex-independent genotype frequencies at the zygote stage are given by 
$Z_{i j, k l, m n}$. First, viability selection occurs. Viabilities are different in the two sexes (see Table 1). Females carrying $P_{1}$ alleles suffer from a fixed viability cost $c_{p}$ (cost of preference). For simplicity, we assume that viability selection at the preference locus is additive (viability of $P_{0} P_{1}$ heterozygotes is $1-\frac{c_{p}}{2}$ ). Likewise, the male trait may come at a viability cost $c_{t} \cdot{ }^{c 1}$ We assume that $T_{1} T_{1}$ homozygotes have a viability $1-c_{t}$ while $T_{0} T_{1}$ heterozyote viability is given by $1-h_{t} c_{t}$ (where $h_{t}$ denotes the dominance coefficient). In the case of a distorter with homozygous lethal effects, ${ }^{c 2} \underline{S}_{1} S_{1}$ individuals have zero viability irrespective of sex. The resulting overall viabilites for males $w_{i j, k l, m n}$ and females $v_{i j, k l, m n}$ are then given as the product of the viability effects at each locus. Based on the zygote frequencies $Z_{i j, k l, m n}$, we can calculate the adult genotype frequencies:

$$
X_{i j, k l, m n}=Z_{i j, k l, m n} \frac{v_{i j, k l, m n}}{\bar{v}}, Y_{o p, q r, s t}=Z_{i j, k l, m n} \frac{w_{i j, k l, m n}}{\bar{w}},
$$

where $\bar{v}$ and $\bar{w}$ denote mean female and male viability, respectively.

In the second step, adults of the present generation mate with each other. Females choose mates according to fixed relative preferences. This relative tendency of a female of $P$-genotype $k l$ to mate with a male of $T$-genotype $o p$ is given by $a_{k l \times o p}$ (see also Table 1). Parameters $h_{p}$ and $h_{a}$ describe dominance effects of preference, where $h_{p}$ defines preference strength of $P_{0} P_{1}$ heterozygote females and $h_{a}$ quantifies preference strength for heterozygote $T_{0} T_{1}$ males. The mating frequency between males of genotype $o p, q r$, st and females of genotype $i j, k l, m n$ is thus

$$
F_{i j, k l, m n \times o p, q r, s t}=X_{i j, k l, m n} Y_{o p, q r, s t} \frac{a_{k l \times o p}}{\overline{a_{o p}}},
$$

where $\overline{a_{o p}}$ is a normalizing constant that ensures that the fertility of a female does not depend on her mate choice.

Given the frequencies of the mating combinations from equation (2), we can now calculate the ${ }^{c 1}$ resulting zygote frequencies $Z_{i j, k l, m n}^{\prime}$ of the next, non-overlapping gen- ${ }^{c 1}$ the eration. Zygote frequencies will depend on segregation distortion $d$ as well as on the recombination rate $r_{U V}$ between loci $U$ and $V\left(r_{T S}, r_{P S}, r_{T P}\right)$. These recombination rates are not independent of each other, i.e. for a given combination of $r_{T S}$ and $r_{P S},{ }^{c 2} r_{T P}=r_{T S}+r_{P S}-2 r_{T S} r_{P S}$. In the case of a sterile distorter, matings involving ${ }^{3} S_{1} S_{1}$ males produce no offspring.

${ }^{c 1}$ Here, $T_{0} T_{1}$ heterozygote fitness is given by dominance coefficient $h_{t}$ (viability of $T_{0} T_{1}$ heterozygotes is $\left.1-h_{t} c_{t}\right)$. ${ }^{c 2} S_{0} S_{0}$ 
All results presented in this manuscript reflect numerical solutions of the system of recurrence equations. Distorter frequencies are usually empirically measured at the adult stage. Allele frequencies in this manuscript were hence recorded at the adult stage. At this stage, we also calculated the standardized linkage disequilibrium $D_{u v}$ between allele $U_{1}$ and $V_{1}$ (at frequencies $u_{1}$ and $v_{1}$ ) defined as (Lewontin, 1964)

$D_{u v}^{\prime}=\frac{D_{u v}}{D_{\max }} \quad$ where $D_{u v}=u v_{1}-u_{1} v_{1}$ and $D_{\max }= \begin{cases}\min \left[u_{0} v_{1}, u_{1} v_{0}\right] & \text { if } D_{u v} \geq 0 \\ \min \left[u_{0} v_{0}, u_{1} v_{1}\right] & \text { if } D_{u v}<0 .\end{cases}$

Here, $u v_{1}$ denotes the frequency of $U_{1} V_{1}$ haplotypes among adult genotypes.

${ }^{c 1}$ For most of the manuscript, we will consider a scenario where wildtype allele $S_{0}$ and the male signal $T_{1}$ are fully linked, i.e. $D_{t s}^{\prime}=-1$ and $r_{T S}=0$. This assumption will be relaxed for the last result section.

\section{Results}

\section{Evolution in the absence of a distorter}

We begin our model analysis by considering sexual selection for a costly male trait in the absence of a distorter locus. The evolutionary outcome strongly depends on whether female preferences are cost-free (Fig. 1A) or whether choosiness is associated with costs (Fig. 1B).

Evolution of cost-free preference In the absence of a distorter, the evolution of cost-free female preferences $\left(c_{p}=0\right)$ has been studied in detail both numerically (Heisler and Curtsinger, 1990) and analytically (Gomulkiewicz and Hastings, 1990; Otto, 1991; Greenspoon and Otto, 2009). The evolutionary dynamics strongly resemble its haploid equivalent, Kirkpatrick's classical model of Fisherian sexual selection (Kuijper et al., 2012). Because there is no direct selection on the preference allele, $p_{1}$ evolves as a correlated response to changes at the trait locus (Fisher process). Evolution at the trait locus is determined by the interplay between natural selection (favouring allele $T_{0}$ ) and sexual selection (favouring allele $T_{1}$ ). Natural and sexual selection balance each other at points that form curves of quasi-equilibria in allele frequency space (the red curves in Fig. 1a); these curves correspond to the lines of equilibria in Kirkpatrick's haploid model (Greenspoon and Otto, 2009). While the line of equilibria is always attracting under haploidy, curves of quasi-equilibria can 
either be repelling (Fig. 1Ai) or attracting (Fig. 1Aii) under diploidy, depending on whether the combination of natural and sexual selection induces net underdominance or net overdominance at the male trait locus (Greenspoon and Otto, 2009).

Evolution of costly preference-the lek paradox Any female preference allele will eventually be selected against and disappear from the population if the slightest costs of choosiness are associated with this allele (Pomiankowski, 1987). Both in the case of a repelling and an attracting curve of quasi-equilibria, evolution at the trait locus will eventually stop because one of the two alleles is fixed (case i) or the polymorphic equilibrium is reached (case ii). At this point, there are no indirect benefits of being choosy because the population is monomorphic ${ }^{\mathrm{cl}}$ at the male trait locus (problem i) or none of the male trait alleles are selectively favoured (problem ii). As a consequence, even small choice costs induces selection against the preference allele and will push it to extinction (Fig. 1B). In the literature, this problem is known as the 'lek paradox’.

\section{The distorter as a target of female preferences}

In the scenario considered above, a costly preference could not evolve because the system evolves to a state where the benefits of choosiness become negligible. The situation may be different if female preferences are targeted at a distorter allele. Distortion may help maintain trait variation despite directional sexual selection (problem i) and confer benefits to choosy females even if trait alleles are at a polymorphic equilibrium (problem ii).

It is unlikely that females base their mate choice directly at the males' genotype at the distorter locus. Instead, female preferences will be based on male traits that may convey information on the presence or absence of distorter alleles. Yet, we will postpone the analysis of such a three-locus scenario (distorter locus, trait locus, preference locus) and first consider the much simpler case where females can directly differentiate between distorter genotypes, or, equivalently, where the trait allele $T_{1}$ is in full linkage to the wildtype allele $S_{0}\left(D_{t s}^{\prime}=-1\right)$ and no recombination between the $T$ and the $S$ locus occurs $\left(r_{T S}=0\right)$. Thus, the model reduces to a diallelic 2-locus system, containing $P_{0}$ and $P_{1}$ alleles at the $P$ locus and $T_{1} S_{0}$ and $T_{0} S_{1}$ haplotypes at the trait/distorter locus (henceforth, we will refer to distorter frequency $s_{1}$ only, where $\left.s_{1}=t_{0}=1-t_{1}\right)$. Because $T_{1}$ alleles only occur together with the wildtype $S_{0}$ allele, a 
female that chooses a $T_{1}$ male will, at the same time, avoid the distorter allele $S_{1}$.

We will first consider an illustrative example of mate choice targeted at a sterile distorter allele based on the parameter values of Fig. 1B. Next, we investigate systematically how evolutionary dynamics are affected by model parameters and the type of distorter. Finally, we explain the various outcomes by means of a simple intuitive argument. This will help us understand four qualitatively different evolutionary outcomes and their parameter dependence.

An illustrative example We start with a situation where females avoid a distorter that is selectively neutral in females and induces sterility in males that are homozygous for the distorter (as in case of the 'sterile $t$ haplotypes' in the house mouse, Lyon (1986)). The evolutionary dynamics of sterile, autosomal distorters in the absence of sexual selection $(p=0)$ have been derived by Dunn and Levene (1961): the distorter is positively selected at the genetic level (segregation distortion) while counterselected at the organismic level (male sterility). The two forces balance at a stable, polymorphic equilibrium given by $\hat{s}_{p=0}=2 d-1$ (see red vertical line Fig. 1C).

Figure $1 \mathrm{C}$ shows the evolutionary dynamics if the costly preference is targeted at a distorter. The parameter values are identical to the two scenarios in Fig. 1B, allowing us to directly compare the evolutionary outcome in the presence and absence of a distorter. The costly preference allele $P_{1}$ now rises to fixation, both in the repelling and attracting scenario. The two factors that previously inhibited the spread of costly preference are now avoided. Firstly, the distorter allele $S_{1}$ is not lost despite directional sexual selection against it (problem i, see Fig. 1B). Sexual selection against the distorter is counteracted by segregation distortion favouring the distorter. Note that selection for distorter alleles $S_{1}$ is particularly strong at low distorter frequencies (van Boven and Weissing, 2001; Weissing and van Boven, 2001). The resulting polymorphism prevents the lek paradox and fuels selection at the preference locus. Secondly, choice is beneficial even if the distorter frequencies are at the polymorphic equilibrium $\hat{s}$ (problem ii, see Fig. 1B). Segregation distortion creates a situation where both $S_{1}$ and $S_{0}$ stably coexist, even though $S_{0} S_{0}, S_{0} S_{1}$ and $S_{1} S_{1}$ males dramatically differ in their individual fitness. The costly preference helps females to avoid the fitness costs of mating with distorter-carrying male.

Dependence of preference frequency on model parameters To systematically explore the parameter conditions that facilitate the evolution of a costly preference 
targeted at a distorter, we calculated evolutionary trajectories for systematically varying levels of preference strength $a$, preference $\operatorname{cost} c_{p}$, and distortion strength $d$. Each model run was started with a low preference frequency $p_{1}=0.01$ and the distorter at equilibrium $\left(s_{1}=\hat{s}_{p=0}\right)$. With these starting conditions, we iterated the recurrence equations until allele frequencies reached equilibrium $\left(\hat{p}_{1}, \hat{s}_{1}\right)$, defined as the point where allele frequency changes became exceedingly small $\left(\Delta p_{1}\right.$ and $\left.\Delta s_{1}<10^{-8}\right)$. For simplicity, we assume that trait costs are absent $\left(c_{t}=0\right)$ and females do not differentiate between $S_{0} S_{1}$ and $S_{1} S_{1}$ males $\left(h_{a}=0\right)$, i.e. they avoid them with same probability (a).

2A shows equilibrium preference frequencies $\hat{p}_{1}$ as a function of $a, c_{p}, d$. Overall, the preference allele can invade and persist in a population for a large spectrum of the parameter space considered, if targeted at a sterile or lethal distorter ${ }^{\mathrm{c}}$ (see Supplementary Text $\mathrm{S} 1$ for evolutionary outcomes if the distorter is lethal). In extreme cases, the preference allele can sustain preference costs as high as $c_{p} \approx 0.4$, i.e. a $40 \%$ viability reduction in choosy females. As one would expect intuitively, higher preference costs $c_{p}$ invariably result in reduced preference frequency. ${ }^{c 2}$ Interestingly, both preference strength $a$ and distortion strength $d$ affect equilibrium preference frequencies in a non-monotonic fashion. Preference frequencies are highest at intermediate values of $a$ and $d$. At low and high levels of $a$ and $d$, the spread of a costly preference is typically limited.

A systematic analysis of parameter dependence To intuitively understand the non-monotonic relationship between model parameters and evolutionary outcomes (Fig. 2A), let us schematically examine two ranges of distorter frequency. Firstly, we specify the range of distorter frequencies that can be attained (at equilibrium) for varying frequencies of the preference allele, denoted as the 'feasible distorter frequency range' $\left[\hat{s}_{p=0}, \hat{s}_{p=1}\right]$ (red shaded areas in Fig. 3). It falls between the distorter equilibrium where preference is absent $\left(\hat{s}_{p=0}\right)$ and the distorter equilibrium where all females in a population are choosy $\left(\hat{s}_{p=1}\right)$. Secondly, we specify the distorter frequency range for which the preference allele is selectively favoured (i.e. where choice benefits outweigh costs), denoted as the 'preference favouring distorter range' $\left[s^{-}, s^{+}\right]$. Preference costs, as implemented in the model, are distorter frequency independent (see yellow line in Fig. 3A). Preference benefits, on the other hand, crucially depend on distorter frequency: if the distorter allele is absent $\left(s_{1}=0\right)$ or fixed $\left(s_{1}=1\right)$ a female will gain 
no benefits from choice. Intermediate distorter frequency confers highest benefits (see green line in Fig. 3A). As a result, the preference allele will only be selectively favoured in the range $\left[s^{-}, s^{+}\right]$(grey shaded area in Fig. 3). The points $s^{-}$and $s^{+}$mark the unstable and stable preference equilibria, respectively, where preference costs and benefits are in balance.

Fig. 3 schematically illustrates the two ranges and how model parameters affect their size and position. The feasible distorter range and preference favouring range can be arranged in seven different ways (scenario 1-7) that correspond to four qualitatively distinct evolutionary outcomes. The evolution of the costly preference allele is limited, whenever the feasible distorter frequencies fall outside the preference favouring range.

If preference strength is very small / ineffective or distortion is strong, preference costs either outweigh benefits for all distorter frequencies (scenario 1, Fig. 2[1]) or the preference favouring range falls completely outside the feasible distorter range (scenario 2, Fig. 2[2]), and the preference allele is lost. At intermediate preference and distorter strength, feasible distorter frequencies are most likely to overlap (at least partly) with the preference favouring range, thereby creating conditions most favourable for costly choice evolution. If unstable equilibrium point $s^{-}$falls inside the feasible distorter range, evolutionary trajectories will depend on whether distorter frequency falls above or below $s^{-}$, resulting in a bistable system with two equilibrium points (scenarios 3 and 4, Fig. 2[3] and [4]). If the costly choice is favoured for all feasible distorter frequencies, it will rise to fixation irrespective of starting frequency (scenario 5, Fig. 2[5]). Interestingly, very strong preferences (and weak distorters) also limit the costly preference evolution, because effective choice typically drive distorters close to extinction, at which point costly choice no longer returns net benefits (as was the case in 'lek paradox' scenarios in the absence of a distorter). The result is either the extinction of the preference (scenario 7, Fig. 2[7]) or damped oscillatory dynamics around stable equilibrium $s^{+}$(scenario 6, Fig. 2[6a,b]). In scenario 6, female choice puses distorter frequencies close to extinction where preference benefits are marginal, which results in a decrease of preference levels. This decrease will, in turn, weaken sexual selection against distorters, allowing $S_{1}$ alleles to increase once again, and the cycle starts anew. 


\section{Recombination between the male signal and the distorter}

So far, we have assumed full linkage between the trait and the distorter locus, thus effectively considering a two-locus system where the distorter allele is a direct target of female mate choice. While this assumption may be realistic for some distorter systems (Williams and Lenington, 1993), potential male signals may be more loosely coupled to the distorter in others (e.g. in stalk-eyed flies). Analyzing the full complexity of the three-locus model is a daunting task. We therefore restricted our analysis to a simpler question: How do the results of the previous section change if we introduce a low level of recombination between the trait locus $T$ and the distorter locus $S$ (i.e. $\left.r_{T S}\right)$ ? To this end, we started at the equilibrium in full linkage $\left(D_{t s}^{\prime}=-1\right)$ and considered the subsequent evolution of the system for a small but positive value of $r_{T S}$. We find that preference alleles disappear from the population already at minimal levels of recombination $\left(r_{T S}=10^{-3}\right.$, see Fig. S2). Recombination will rapidly produce an increasing number of $T_{1} S_{1}$ haplotypes that are favoured both by sexual selection and distortion. Because choosy females increasingly mate with distorter carrying males, female choice will no longer confer fitness benefits and thus be lost. We examined all parameter combinations shown in Fig. 2 in this manner, and find that conclusion is representative for the whole parameter space explored in this study. Hence, the successful evolution of the costly preference breaks down even at marginal recombination rates between the male trait and the distorter.

\section{Discussion}

We have demonstrated that female choice for distorter-free males can spread and persist in a population even if mate choice is associated with considerable direct fitness costs. This is in contrast to classical models of sexual selection where preference costs typically result in the loss of female preference (Kuijper et al., 2012). Two key components of the distorter enable spread and maintenance of the costly female choice allele. The spread is a consequence of the large benefits associated with avoiding carriers of distorter alleles. The maintenance results from the fact that segregation distortion helps preserve male trait variation despite directional sexual selection. The balance of gene-level selection in favour and individual-level selection against the distorter alleles keeps allele frequencies at the distorter locus in a firm polymorphic state, thus avoiding the lek paradox that often hampers the maintenance of costly mate choice. Akin 
to previous resolutions (Kotiaho et al., 2008), the present model proposes a mechanism (distortion) that maintains trait variation in the face of directional sexual selection. Our model has also identified several factors that limit the evolution of the costly preference allele. Interestingly, we find that preference evolution is limited if the distorter is very strong or if the preference allele induces strong preferences. In the latter case, the lek paradox prevails. Moreover, we show that the costly preference can only spread in the presence of a signal that reliably indicates a male's distorter genotype. Accordingly, already the smallest degree of recombination between a male signal and the distorter will result in the disappearance of the costly preference.

Our findings are consistent with the few previous models addressing mate choice evolution in the presence of distorters, all focusing on different types of sex-linked distorters (Lande and Wilkinson, 1999; Reinhold et al., 1999; Randerson et al., 2000). In the case of sex-linked distortion, choice benefits stem from the fact that mating with a distorter-free partner will result in offspring of even sex ratio. Since the sex ratio of populations harbouring sex-linked distorters is strongly biased, producing offspring of the rarer sex convey a selective advantage as individuals of the rarer sex have a higher reproductive value (Pen and Weissing, 2001). The conclusions are similar to the ones presented here: cost-free (Lande and Wilkinson, 1999) and costly (Randerson et al., 2000) mate choice for distorter/male-killer-free mates can stably persist. Mate choice for drive suppression, on the other hand, seems not beneficial (Reinhold et al., 1999). Despite these similarities, there may be quantitative differences between autosomal and sex-linked distorters. ${ }^{c 1}$

Reliable indicators of distortion Preference benefits of female choice are only guaranteed if the male trait is a reliable indicator of the genetic status at the distorter locus. In line with Lande and Wilkinson (1999), we found that ${ }^{{ }^{2} 2}$ even small recombination rates between trait and distorter inhibit the spread of the choice allele as they erode the reliability of the signal and hence benefits of choice. Given this restrictive prerequisite, one may conclude that our model can explain the presence of a costly

${ }^{c 1}$ In sex-linked distorters, relatively weak levels of sexual selection appear sufficient to drive the distorter to extinction. With the autosomal distorter considered here, strong sexual selection is needed to oppose distortion, allowing for a larger range of favourable conditions to the maintenance of a costly choice. c2 already preference for distorter-free mates in only few real-world systems. However, we see two scenarios in which the model can be relevant. Full linkage between a sexually selected trait and a distorter is possible if recombination between the loci is suppressed or the distorter itself is the signal (i.e. the signal is a pleiotropic effect of the distorter). Both scenarios may be relevant at different stages of mating process. 
Premating stage In the case of premating choice, a scenario where the distorter itself is the target of female mate choice seems unlikely. In fact, distorters typically have no or little effects on the external phenotype (Burt and Trivers, 2006) that may serve as signals for premating mate choice. Suppression of recombination between the distorter and a trait 'recognizable' to females will thus be required. Interestingly, suppression of recombination is an essential part of distorter systems as the effects of segregation distortion hinge upon the interaction of several genes (but also see van Boven and Weissing (2000)). In fact, recombination has been proposed as a way for an organism to avoid selfish action of groups of linked genes by decoupling possible alliances (Leigh, 1971). So what makes a distorter effective in the first place, suppression of recombination, may render them at the same time vulnerable to negative sexual selection. Through the lack of recombination, there is a chance that the distorter will be bound to a gene with phenotypic effects recognizable to females, thereby allowing mate choice against it. The $t$ haplotype in house mice, for example, consists of about 300 genes linked to each other through four chromosomal inversions (Burt and Trivers, 2006). Among these genes are several major histocompatibility complex (MHC) loci, that have been proposed as signals mediating mate choice (Milinski, 2006). In a study on a wild house mouse population, Lindholm et al. (2013) have shown that $t$ haplotypes were associated with a unique and exclusive MHC allele. There is mixed evidence for mate choice in the $t$ haplotype system. It has been detected in some (Lenington et al., 1992), but not all populations (Manser et al., 2015; Sutter and Lindholm, 2016), and the role of MHC remains controversial (Lenington et al., 1988). Overall, there is only limited evidence for mating preferences in connection with distorters (Wilkinson et al., 1998; Wedell, 2013; Price et al., 2012). Price

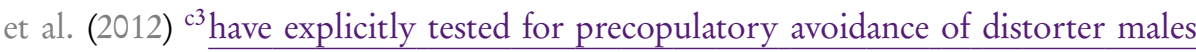
in Drosophila psendoobscura but did not any evidence in a series of lab experiments. The requirement of a signal accidentally trapped in the distorter's linkage group may explain why ${ }^{c 4}$ premating choice is so rare.

Postmating stage Females can also avoid fertilization by distorter-carrying males at the postmating stage. The mechanisms underlying segregation distortion typically lead to lower sperm number and/or lower sperm quality. As a result, distortercarrying males are often compromised in their sperm competitive ability (Price and Wedell, 2008). Females may capitalize on this fact by mating with multiple males 
(polyandry), thereby avoiding fertilization by distorter-males (Haig and Bergstrom, 1995). This is interesting in the context of the model presented here, because the phenotype causing non-random ${ }^{c 1}$ fertilization, reduced sperm competitiveness, is a pleiotropic effect of the distorter itself. Hence, no suppression of recombination between a signal and the distorter is needed for the evolution of polyandry. This may explain why empirical evidence for polyandry as a female counterstrategy against distorters is far more abundant than premating choice. ${ }^{\mathrm{c} 2}$ Despite parallels, sperm competition differs from the choice model presented here in important ways. Precmating choice here is based on a fixed relative preference, i.e. the strength of preference is independent of distorter frequency, whereas in the context of polyandry, sperm competition only matters if both male types are present in the sample of males a female mates with. The effectiveness of polyandry will hence be a function of distorter frequency, i.e. decrease with increasing distorter frequency (as distorter males are more likely to compete against other distorter males if frequencies are high). ${ }^{c 3}$ In a recent study, Holman et al. (2015) investigated this scenario in the context of a sex-linked distorter. ${ }^{c 4}$ Akin to the results presented here, they found that polyandry can evolve and be stably maintained if the distorter is stabilised at an intermediate frequency by negative frequency dependent selection. Further studies are required to investigate ${ }^{55}$ whether their findings translate to autosomal distortion systems.

Maintaining Distorter Alleles Our model demonstrates that a costly mate choice can only successfully evolve if distorter frequencies are kept at intermediate frequencies, where the preference allele is selectively favoured. The successful spread of the preference allele is hence the result of a delicate balancing act. Any selective force that pushes distorter frequencies to one or the other extreme of the distorter frequency spectrum will limit the evolution of costly choice. Accordingly, we found that very strong or weak levels of segregation distortion hamper the spread of the preference allele. Similarly, a costly mate choice can only be maintained efficiently if the preference is of intermediate strength. If directional sexual selection is strong, it may override the distorter's capacity to create new male trait variance. In this case, the lek paradox prevails. Once choosy females have successfully removed most distorters from the population, i.e. male variation has expired, preference costs again start to outweigh preference benefits, just as in our original scenario without a distorter (where already marginal levels of preference are sufficient to run into that problem, see Fig. 1B). Pref-

c3 A 'best-of-N' mechanism, where females choose a male of a given male subsample, may be a more adequate depiction of polyandry.

c4 They found that the evolution of costly polyandry can indeed evolve in circumstances where the sex-distorter is associated with additional organismal fitness costs.

${ }^{c 5}$ how such an alteration in the mate choice mechanism affects the evolutionary outcomes in 
erence frequency will then stabilize at a lower level that allows for enough male trait variation to keep benefits and costs of choice in balance (scenario 6). In other words, costly mate choice for Mendelian segregation will only escape the lek problem in a given spectrum of preference strength. At the lower end of the spectrum, choice is not effective and benefits of choice are hence limited. At the upper end of the spectrum, sexual selection is -once more- too strong to maintain male trait variation. It is unclear if the levels of preference strength needed for this second effect are biologically relevant. However, the question whether there is an optimal level of preference strength is an interesting theoretical question in itself, especially considering the nonstraightforward relationship between preference strength and equilibrium preference frequency.

Does Mate Choice Explain Distorter Frequencies? So far, we have largely focused on the distorter's influence on the sexual selection process. However, we can also ask how costly female choice affects distorter dynamics. Accounting for distorter frequencies in wild populations is a long standing focus of evolutionary theory (Burt and Trivers, 2006). Depending on its strength, female mate choice may be an important determinant of distorter frequency (e.g. Manser et al. (2011)). ${ }^{c 1}$ Akin to molecular suppressors of distortion proposed elsewhere, female mate choice can be seen as a behavioural mechanism to reduce drive frequency by creating selection against individuals that carry a distorter. By undermining the spread of the selfish distorter, female choice may help to maintain harmony at the genomic level. However, our current model suggests that this mechanism will only be successful to a certain degree, at least as long as female choice is costly and drift effects are negligible. The scenario where the lek paradox prevails as a consequence of strong directional sexual selection (scenario 2) makes clear that female choice will never completely remove the distorter. As soon as mate choice is effective in removing distorter alleles, benefits of choice fade, allowing the distorter back in. Intriguingly, this may provide an explanation for a problem known as the as the low $t$ frequency paradox in house mice. In the $t$ haplotype system in house mice, $t$ frequencies in wild populations are usually at low (lower than expected from distortion and lethality only) but stable levels (Ardlie, 1998; van Boven and Weissing, 1999). Costly female choice may explain why $t$ frequencies are lower than expected, yet stably prevail in populations. 
A general mechanism for the evolution of costly mate choice? The presented model demonstrates that segregation distorters can greatly facilitate the evolution of female choice, even if such a choice is associated with substantial fitness costs. We can only speculate about the importance of distorter systems for the evolution of female choice in general. Selfish genetic elements are considered a ubiquitous feature of life (Burt and Trivers, 2006). However, the abundance of autosomal distorter systems considered here, particularly among animals, is largely unknown. The covert action of distorters make detection and identification inherently difficult. It is not surprising that the best known distorter systems were both found in two of the beststudied model organisms ( $t$ haplotype in the house mouse and Segregation Distorter in Drosophila). Deviations from Mendelian inheritance are occasionally reported in other species, but the causes of such biased inheritance is often unknown (Burt and Trivers, 2006). In both known cases, segregation distortion is relatively effective $(d \approx 0.9)$. It is not known whether this feature is representative of distorter systems in general or whether it is the result of a detection bias (as weaker distorters are more difficult to discover). Our model suggests that a weak distorter's capacity to promote female mate choice is reduced, because weak distortion easily results in distorter equilibria outside the preference favouring range (scenarios 6 and 7). However, if not only distortion is weaker, but also its selective effects on the organism (here, distorters result in male sterility or homozygote lethality), distorter equilibria may well shift back into the preference favourable range. In any case, the present model shows the action of distorters, usually hidden from sight, may play an important role in driving the evolution of costly female choice, both at a pre- and postmating stage.

Acknowledgements We thank Andrew Pomiankowski, Luke Holman, and an anonymous reviewer for his helpful feedback on a previous version of this manuscript. This work was funded by the Forschungskredit of the University of Zürich, the Claraz Foundation, and the Swiss National Science Foundation (SNSF: 310030M-138389).

Approximate word count: 5,400

Ardlie, K., 1998. Putting the brake on drive: meiotic drive of $t$ haplotypes in natural populations of mice. Trends in Genetics 14:189-193.

Atlan, A., D. Joly, C. Capillon, and C. Montchamp-Moreau, 2004. Sex-ratio distorter of Drosophila simulans reduces male productivity and sperm competition ability. Journal of Evolutionary Biology 17:744.

van Boven, M. and F. J. Weissing, 1999. Segretation distortion in a deme-structured population: 
opposing demands of gene, individual and group selection. Journal of Evolutionary Biology 12:80-93.

2000. Evolution at the mouse $t$ complex: why is the $t$ haplotype preserved as an integral unit? Evolution 54:1795-1808.

-_-, 2001. Competition at the mouse $t$ complex: Rare alleles are inherently favored. Theoretical Population Biology 60:343-358.

Brooks, R. C. and S. C. Griffith, 2010. Mate choice. in D. Westneat, C. Fox, and C. W. Fox, eds. Evolutionary behavioral ecology. Oxford University Press.

Bruck, D., 1957. Male segregation ratio advantage as a factor in maintaining lethal alleles in wild populations of house mice. Proceedings of the National Academy of Sciences 43:152-158.

Burt, A. and R. Trivers, 2006. Genes in conflict: The biology of selfish genetic elements. Belknap Press, Cambridge.

Charlat, S., M. Reuter, E. A. Dyson, E. A. Hornett, A. Duplouy, N. Davies, G. K. Roderick, N. Wedell, and G. D. Hurst, 2007. Male-killing bacteria trigger a cycle of increasing male fatigue and female promiscuity. Current Biology 17:273-277.

Cotton, A., M. Földvári, S. Cotton, and A. Pomiankowski, 2014. Male eyespan size is associated with meiotic drive in wild stalk-eyed flies (Teleopsis dalmanni). Heredity 112:363-369.

Dunn, L. and H. Levene, 1961. Population dynamics of a variant $t$-allele in a confined population of wild house mice. Evolution 15:385-393.

Gomulkiewicz, R. and A. Hastings, 1990. Ploidy and evolution by sexual selection: a comparison of haploid and diploid female choice models near fixation equilibria. Evolution 44:757-770.

Greenspoon, P. and S. Otto, 2009. Evolution by fisherian sexual selection in diploids. Evolution 63:1076-1083.

Haig, D. and C. Bergstrom, 1995. Multiple mating, sperm competition and meiotic drive. Journal of Evolutionary Biology 8:265-282.

Heisler, I. L. and J. W. Curtsinger, 1990. Dynamics of sexual selection in diploid populations. Evolution 44:1164-1176.

Holman, L., T. A. R. Price, N. Wedell, and H. Kokko, 2015. Coevolutionary dynamics of polyandry and sex-linked meiotic drive. Evolution 69:709-720. URL http://dx.doi. org/10.1111/evo.12595.

Kirkpatrick, M. and M. Ryan, 1991. The evolution of mating preferences and the paradox of the lek. Nature 350:33-38.

Kotiaho, J., N. LeBas, M. Puurtinen, and J. Tomkins, 2008. On female choice, heterozygosity and the lek paradox. Animal Behaviour 75:1-3.

Kotiaho, J. S., L. W. Simmons, and J. L. Tomkins, 2001. Towards a resolution of the lek paradox. Nature 410:684-686.

Kuijper, B., I. Pen, and F. J. Weissing, 2012. A guide to sexual selection theory. Annual Review of Ecology, Evolution, and Systematics 43:287-311.

Lande, R. and G. Wilkinson, 1999. Models of sex-ratio meiotic drive and sexual selection in stalk-eyed flies. Genetical Research 74:245-253.

Leigh, E., 1971. Adaptation and diversity. Freeman \& Cooper, San Francisco US.

Lenington, S., C. Coopersmith, and J. Williams, 1992. Genetic basis of mating preferences in wild house mice. Integrative and Comparative Biology 32:40-47. 
Lenington, S., K. Egide, and J. Williams, 1988. Analysis of a genetic recognition system in wild house mice. Behaviour Genetics 18:549-564.

Lewontin, R., 1964. The interaction of selection and linkage. I. General considerations; heterotic models. Genetics 49:49.

Lindholm, A. K., K. Musolf, A. Weidt, and B. König, 2013. Mate choice for genetic compatibility in the house mouse. Ecology and Evolution 3:1231-1247.

Lyon, M. F., 1986. Male sterility of the mouse $t$-complex is due to homozygosity of the distorter genes. Cell 44:357-363.

Manser, A., B. König, and A. K. Lindholm, 2015. Female house mice avoid fertilization by $\mathrm{t}$ haplotype incompatible males in a mate choice experiment. Journal of Evolutionary Biology 28:54-64.

Manser, A., A. K. Lindholm, B. König, and H. C. Bagheri, 2011. Polyandry and the decrease of a selfish genetic element in a wild house mouse population. Evolution 65:2435-2447.

Milinski, M., 2006. The major histocompatibility complex, sexual selection, and mate choice. Annual Review of Ecology, Evolution, and Systematics 37:159-186.

Miller, W. J., L. Ehrman, and D. Schneider, 2010. Infectious speciation revisited: impact of symbiont-depletion on female fitness and mating behavior of Drosophila paulistorum. PLoS Pathogens 6:e1001214.

Otto, S., 1991. On evolution under sexual and viability selection: a two-locus diploid model. Evolution Pp. 1443-1457.

Pen, I. and F. Weissing, 2001. Sexual selection and the sex ratio: an ESS analysis. Selection 1:111-122.

Pomiankowski, A., 1987. The costs of choice in sexual selection. Journal of Theoretical Biology 128:195-218.

Pomiankowski, A., Y. Iwasa, and S. Nee, 1991. The evolution of costly mate preferences I. Fisher and biased mutation. Evolution 45:1422-1430.

Pomiankowski, A. and A. Moller, 1995. A resolution of the lek paradox. Proceedings of the Royal Society of London. Series B: Biological Sciences 260:21-29.

Price, T., D. Hodgson, Z. Lewis, G. Hurst, and N. Wedell, 2008. Selfish genetic elements promote polyandry in a fly. Science 332:1241-1243.

Price, T., Z. Lewis, D. Smith, G. Hurst, and N. Wedell, 2012. No evidence of mate discrimination against males carrying a sex ratio distorter in drosophila pseudoobscura. Behavioral Ecology and Sociobiology 66:561-568.

Price, T. and N. Wedell, 2008. Selfish genetic elements and sexual selection: their impact on male fertility. Genetica 134:99-111.

Randerson, J. P., F. M. Jiggins, and L. D. Hurst, 2000. Male killing can select for male mate choice: a novel solution to the paradox of the lek. Proceedings of the Royal Society of London. Series B: Biological Sciences 267:867-874.

Reinhold, K., L. Engqvist, B. Misof, and J. Kurtz, 1999. Meiotic drive and evolution of female choice. Proceedings of the Royal Society of London. Series B: Biological Sciences 266:13411345.

Sutter, A. and A. K. Lindholm, 2016. No evidence for female discrimination against male house mice carrying a selfish genetic element. Current Zoology URL http://cz. oxfordjournals .org/content/early/2016/06/28/cz. zow063. 
Tomkins, J. L., J. Radwan, J. S. Kotiaho, and T. Tregenza, 2004. Genic capture and resolving the lek paradox. Trends in Ecology \& Evolution 19:323-328.

Wedell, N., 2013. The dynamic relationship between polyandry and selfish genetic elements. Philosophical Transactions of the Royal Society B: Biological Sciences 368:1-10.

Weissing, F. J. and M. van Boven, 2001. Selection and segregation distortion in a sexdifferentiated population. Theoretical Population Biology 60:327-341.

Wilkinson, G., D. Presgraves, and L. Crymes, 1998. Male eye span in stalk-eyed flies indicates genetic quality by meiotic drive suppression. Nature 391:276-279.

Williams, J. and S. Lenington, 1993. Factors modulating preferences of female house mice for males differing in $t$-complex genotype: role of $t$-complex genotype, genetic background, and estrous condition of females. Behavior Genetics 23:51-58.

Zeh, J. and D. Zeh, 1997. The evolution of polyandry II: post-copulatory defences against genetic incompatibility. Proceedings of the Royal Society B: Biological Sciences 264:69-75. 
Table 1. Overview over the three loci and the parameters used in the model. Sex symbol in brackets indicate the sex in which the given property is expressed.

\begin{tabular}{|c|c|c|c|c|}
\hline Trait locus $T$ & & $T_{0} T_{0}$ & $T_{0} T_{1}$ & $T_{1} T_{1}$ \\
\hline Viability (o") & & 1 & $1-h_{t} c_{t}$ & $1-c_{t}$ \\
\hline Preference locus $P$ & & $P_{0} P_{0}$ & $P_{0} P_{1}$ & $P_{1} P_{1}$ \\
\hline \multirow[t]{3}{*}{ Preferences (\$) } & $T_{0} T_{0}$ & 1 & 1 & 1 \\
\hline & $T_{0} T_{1}$ & 1 & $1+b_{a} b_{p} a$ & $1+b_{a} a$ \\
\hline & $T_{1} T_{1}$ & 1 & $1+h_{p} a$ & $1+a$ \\
\hline Viability (o) & & 1 & $1-c_{p} / 2$ & $1-c_{p}$ \\
\hline Segregation locus $S$ & & $S_{0} S_{0}$ & $S_{0} S_{1}$ & $S_{1} S_{1}$ \\
\hline either: Viability (ه"థ) & & 1 & 1 & 0 \\
\hline or: Fertility (o") & & 1 & 1 & 0 \\
\hline Segregation ratio $\left(\sigma^{\prime}\right)$ & & 0 & $d$ & 1 \\
\hline
\end{tabular}



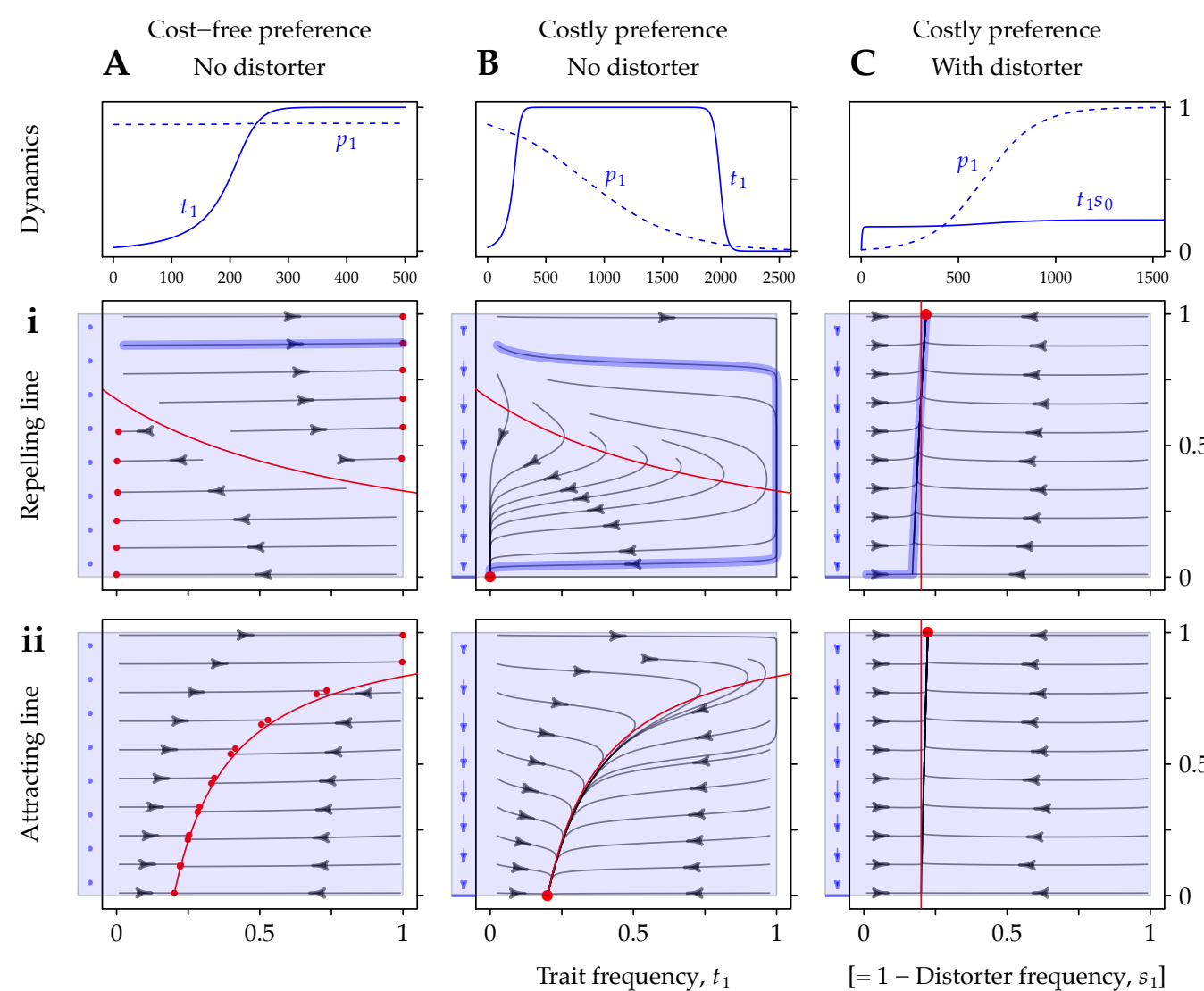

Figure 1. Joint evolution of trait $t_{1}$ and preference $p_{1}$ alleles in the absence (A-B) and presence (C) of a distorter. Center row panels (i) illustrate a scenario of a repelling line of quasi-equilibria, lower row panels (ii) a scenario an attracting line of quasi-equilibria (indicated by the red lines, based on Greenspoon and Otto (2009)). Top panels follow the allele frequency dynamics of a specific evolutionary trajectory of scenario i over time (shaded in blue). In $\mathrm{A}$, the preference is cost-free (parameter values for scenario i: $a=0.4, h_{a}=0.5, h_{p}=0.3, p_{c}=0, c_{t}=0.15$, $h_{t}=0.5, r_{P T}=0.5$; parameter values for scenario ii: $a=0.4, h_{a}=0.5, h_{p}=0, p_{c}=0, c_{t}=0.2$, $\left.h_{t}=-1 / 3, r_{P T}=0.5\right)$. In $\mathrm{B}$, a preference cost $c_{p}=0.005$ is added, resulting in the collapse of the quasi-neutral curves to a single, attracting point, where the preference allele is absent. In $\mathrm{C}$, the preference is targeted at a sterile distorter $(d=0.9$, the remaining parameter values are identical to B). Now, the preference allele rises to fixation. The red vertical line indicates the distorter equilibrium in the absence of preference $\left(\hat{s}_{p=0}\right)$. The blue arrows and shades illustrate selection on preference alleles in the absence of a distorter/male trait $\left(p_{1}=0\right)$. The red dots indicate the end points (equilibria) of each evolutionary trajectory. 
A Preference strength, $a$

Segregation distortion, $d$
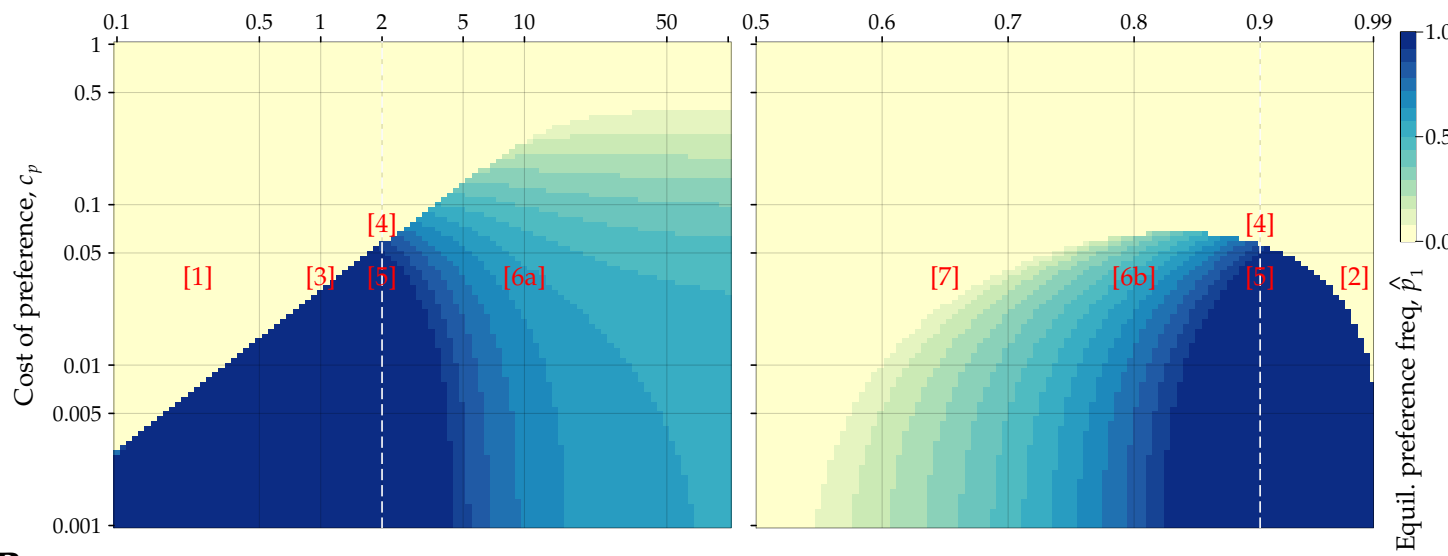

B

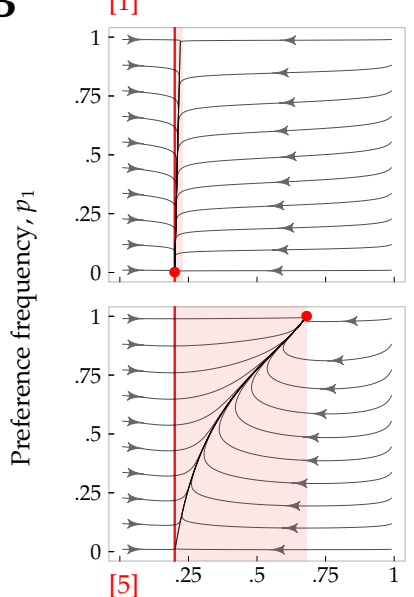

[2]

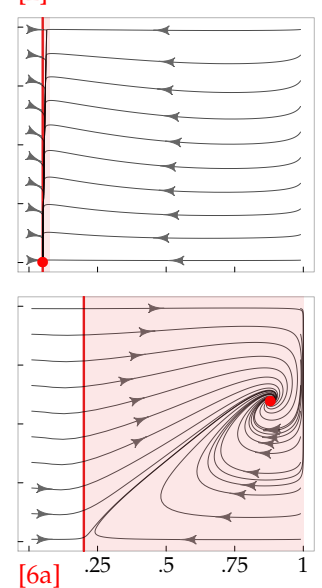

[3]
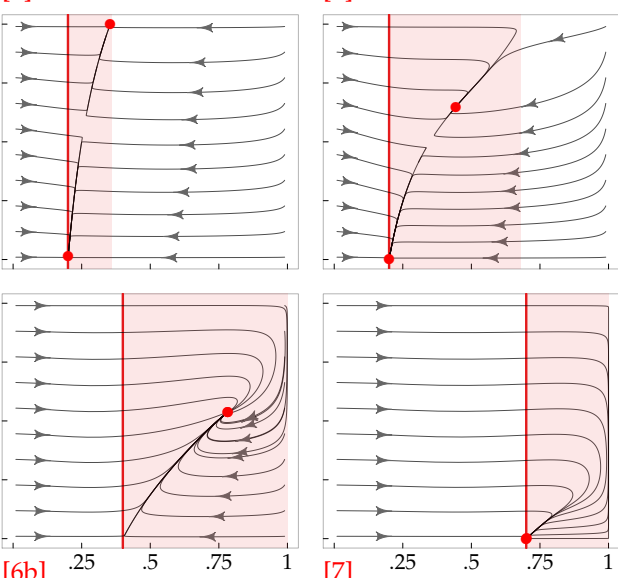

Trait frequency, $t_{1}=1-$ Distorter frequency, $s_{1}$

Figure 2. A. Equilibrium preference frequencies $\hat{p}_{1}$ of a preference allele targeted at a sterile distorter in relation to preference strength $(a)$, preference cost $\left(c_{p}\right)$ and distorter strength $(d)$. Preference strength $a$ and $\operatorname{cost} c_{p}$ are shown on $\log _{10}$-scale. Left panels are based on a distorter strength of $d=0.9$, right panels on a preference strength of $a=2$, with the vertical dotted lines indicating the location where phase-plots intersect. Red numerals (1-7) depict parameter combinations that correspond to scenarios 1-7 that are schematically summarised in Figure 3 (scenario 6 occurs twice). B. Evolutionary trajectories of distorter $s_{1}$ and preference $p_{1}$ alleles of the respective parameter combination/scenario. The red vertical line indicates the distorter equilibrium in the absence of preference $\hat{s}_{p=0}$. The red shaded area denotes the feasible distorter range. The red dots correspond to the end point of each evolutionary trajectory. The red dots correspond to the end point of each evolutionary trajectory ( equilibrium). Remaining parameter values: $c_{t}=0, h_{p}=0.5, h_{a}=0, r_{P S}=r_{P T}=0.5$. 


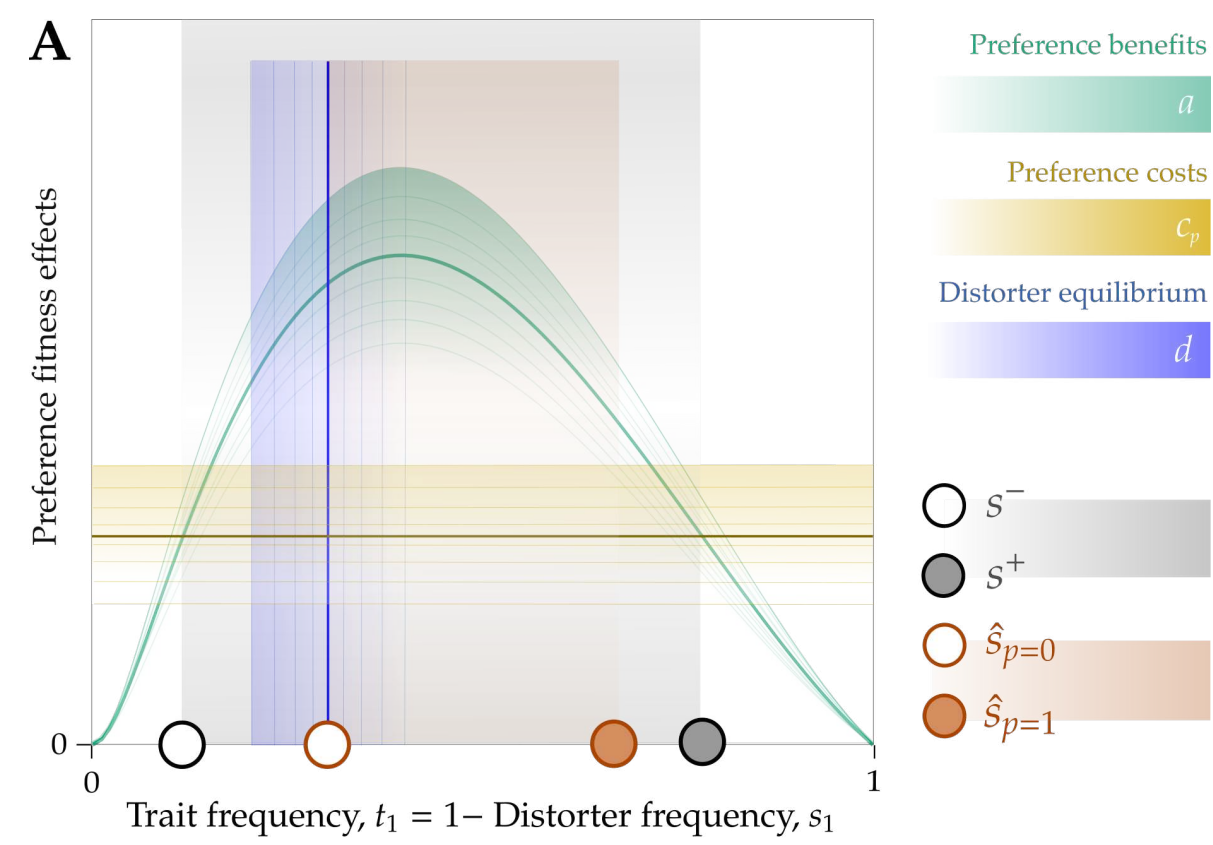

\begin{tabular}{|c|c|c|c|c|c|}
\hline Scenario & Condition & & $\hat{p}_{1}$ & $\hat{s}_{1}$ & Parameters \\
\hline 1 & & & 0 & 0 & $d$ \\
\hline 2 & & 0 & 0 & 0 & \\
\hline 3 & 0 & 0 & 0 or 1 & Oor $\bigcirc$ & \\
\hline 4 & 00 & & 0 or $[0,1]$ & Oor $\bigcirc$ & \\
\hline 5 & 0 & 0 & 1 & 0 & \\
\hline 6 & $0 \quad 0$ & & {$[0,1]$} & 0 & \\
\hline 7 & 0 & & 0 & 0 & $a$ \\
\hline
\end{tabular}

Figure 3. A. Schematic illustration of the feasible distorter frequency range (area shaded in red) and the preference favouring distorter range (area shaded in grey) for intermediate levels of preference strength $a$, preference costs $c_{p}$, and distorter strength $d$ (indicated by green, yellow, and blue lines, respectively, corresponding to scenario 5). The feasible distorter range corresponds to the spectrum of the distorter equilibria, ranging from the distorter equilibrium where preference is absent $\hat{s}_{p=0}$ (open red dot) to the distorter equilibrium where all females express a preference $\hat{s}_{p=1}$ (closed red dot). PARAMETER DEPENDENCE: The blue shading illustrates how the position of $\hat{s}_{p=0}$ depends on the levels of distorter strength $d$ (with darker shades represening higher $d$ levels). The position of $\hat{s}_{p=1}$ will be a function of both preference and distorter strength $a, d$. The preference favouring distorter range specifies the distorter frequency spectrum where the preference allele is selectively favoured, i.e. where preference benefits outweigh preference costs. PARAMETER DEPENDENCE: The yellow shading illustrates the effect of preference costs levels $c_{p}$. Note that preference costs are distorter frequency independent. Green shading illustrate the effect preference strength $a$. The points where the cost and benefit line intersect, i.e. where preference costs and benefits are in balance, correspond to unstable and stable preference equilibria $s^{-}$(open black dot) and $s^{+}$(closed black dot), respectively. B. Overview over the seven possible scenarios, the four qualitatively different evolutionary outcomes $\left(\hat{p}_{1}, \hat{s}_{1}\right)$, and their parameter dependence. The second column schematically illustrates the relative position of the feasible distorter range (coloured in red) and the preference favouring range (colored in grey). 
598 Supplementary Material

599 S1 Evolutionary Dynamics if the distorter is lethal

600 The two different distorter types (sterile vs. lethal) generate only minor, qualitative

601 differences in the evolutionary outcome (see Fig. S1 and 2). As is the case with sterile

602 distorters, recessive lethal distorters induce a stable, polymorphic equilibrium given

603 by $\hat{s}_{p=0}=\frac{1}{2}-\frac{\sqrt{2 d-1}}{2 d}$ (Bruck, 1957). If at all, the range of parameter values allowing

604 the spread of the costly preference allele $P_{1}$ is slightly smaller in ${ }^{c 1}$ the case of a lethal

c1 Text added. distorter when compared with sterile distorters. This can be understood if one considers that the female benefits of avoiding distorter carriers are slightly different for lethal and sterile distorters. In the case of a lethal distorter, the benefits are straightforward: a female avoiding distorter-carrying males prevents lethality in her progeny. In the case of sterile distorters - at least as it is implemented in the model here- benefits are twofold. First and foremost, a choosy female avoids $S_{1} S_{1}$ homozygotes and hence complete failure of reproduction due to male sterility. Second, she avoids $S_{0} S_{1}$ heterozygote males, which would render a potentially large proportion (depending on her own $S$ genotype) of her male offspring sterile. It is this twofold advantage that may explain why costly preferences targeted at a sterile distorter evolve under a slightly broader parameter range. 

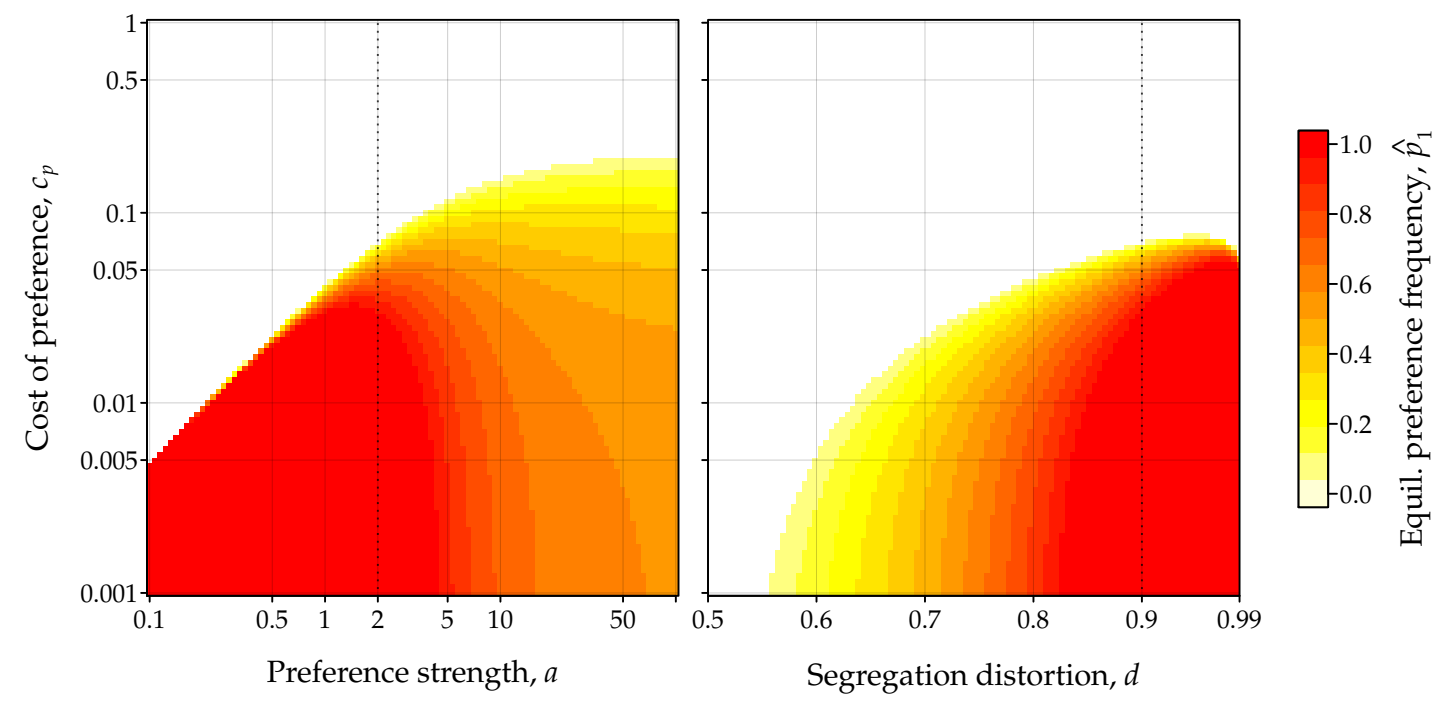

Figure S1. Equilibrium preference frequencies $\hat{p}_{1}$ of a preference allele targeted at a lethal distorter in relation to preference strength $(a)$, preference cost $\left(c_{p}\right)$ and distorter strength $(d)$. Preference strength $a$ and $\operatorname{cost} c_{p}$ are shown on a $\log _{10}$-scale. Left panels are based on a distorter strength of $d=0.9$, right panels on a preference strength of $a=2$, with the vertical dotted lines indicating the location where phase-plots intersect. The figure is identical to the top panel shown for sterile distorter in Fig 2. Remaining parameter values: $c_{t}=0, h_{p}=0.5, h_{a}=0$, $r_{P S}=r_{P T}=0.5$. 

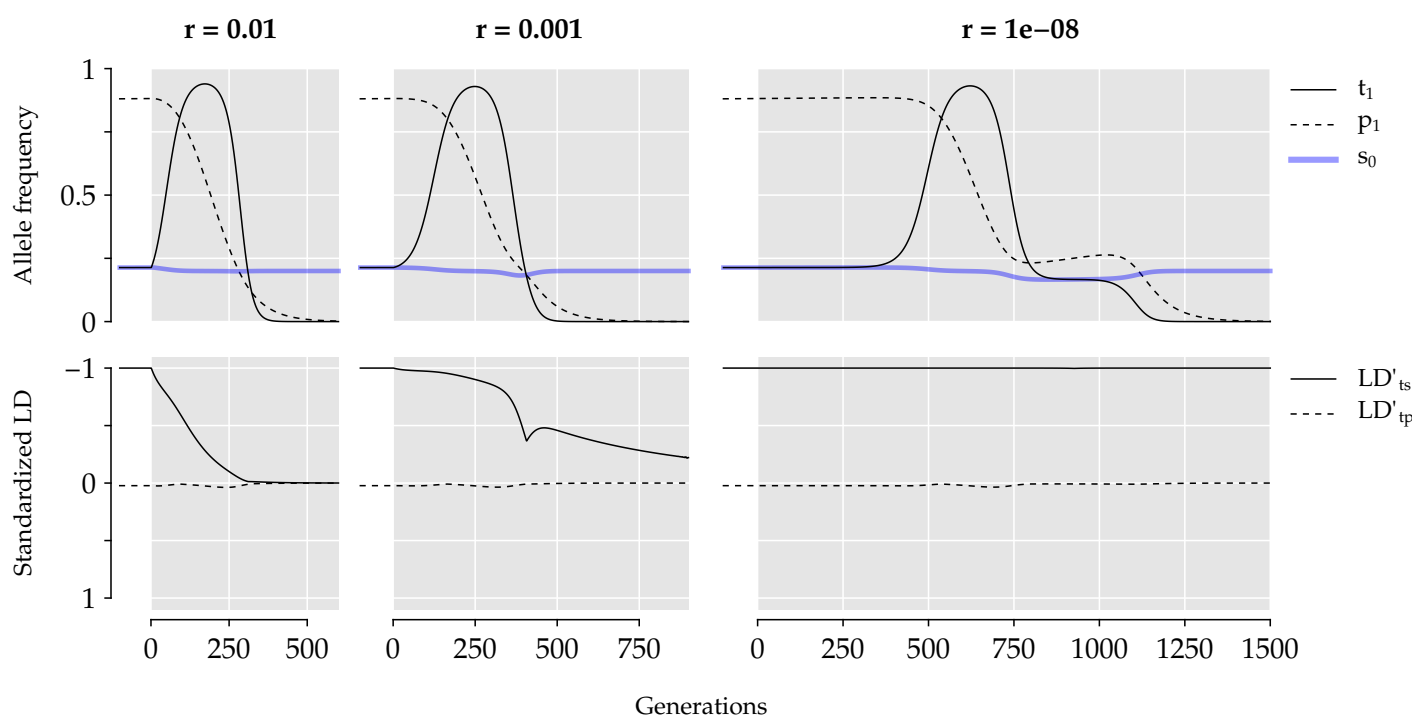

Figure S2. Disappearance of female preference for fair Mendelian segregation as a result of recombination between the trait and the distorter locus. Three different levels of recombination between trait and distorter are shown: $r_{T S}=\left[10^{-2}, 10^{-3}, 10^{-8}\right]$. Upper panels show allele frequencies (trait $t_{1}$, preference $p_{1}$ and non-driving allele $s_{0}$ ) over time. The lower panels depict standardized linkage disequilibria $\left(D_{t s}^{\prime}, D_{t p}^{\prime}\right)$. At first, allele frequencies are fully linked and at equilibrium. At generation 0 (grey shaded area), recombination between distorter and trait is introduced. The resulting dynamics strongly resemble the case without a distorter (Fig. 1C), the costly preference ceases within a short number of generations (even at marginal recombination rates). Remaining parameter values: $a=0.6, h_{p}=0.4, b_{a}=0.5, c_{p}=0.03, c_{t}=0.25$, $d=0.9, r_{P S}=0.5$. 